Research Article

\title{
Control Strategies to Curtail Transmission of COVID-19
}

\author{
Nita H. Shah $(\mathbb{D}$, Ankush H. Suthar, and Ekta N. Jayswal \\ Department of Mathematics, Gujarat University, Ahmedabad 380009, Gujarat, India \\ Correspondence should be addressed to Nita H. Shah; nitahshah@gmail.com \\ Received 30 March 2020; Revised 24 April 2020; Accepted 27 April 2020; Published 21 May 2020 \\ Academic Editor: Vladimir V. Mityushev \\ Copyright $\odot 2020 \mathrm{Nita}$ H. Shah et al. This is an open access article distributed under the Creative Commons Attribution License, \\ which permits unrestricted use, distribution, and reproduction in any medium, provided the original work is properly cited. \\ On 11 March 2020, the World Health Organization declared the outbreak of severe acute respiratory syndrome coronavirus 2 \\ (SARS-Cov-2) a pandemic and a Public Health Emergency of International Concern. As of 29 March 2020, coronavirus disease \\ 2019 (COVID-19) has affected 199 countries and territories, resulting in 683,536 positive cases and causing 32,139 deaths. The \\ pandemic has the potential to become extremely destructive globally if not treated seriously. In this study, we propose a \\ generalized SEIR model of COVID-19 to study the behaviour of its transmission under different control strategies. In the model, \\ all possible cases of human-to-human transmission are considered and its reproduction number is formulated to analyse the \\ accurate transmission dynamics of the coronavirus outbreak. Optimal control theory is applied to the model to demonstrate the \\ impact of various intervention strategies, including voluntary quarantine, isolation of infected individuals, improving an in- \\ dividual's immunity, and hospitalization. In addition, the effect of control strategies on the model is analysed graphically by \\ simulating the model numerically.
}

\section{Introduction}

In December 2019, the pandemic outbreak of coronavirus disease 2019 (COVID-19) drew intense attention not only within China but internationally [1]. Doctors and scientists tested previously developed drugs to treat infected patients, but these have so far been unsuccessful. In March 2020, the World Health Organization (WHO) declared the outbreak of the highly contagious COVID-19 as a global pandemic [2]. To control human-to-human virus transmission, the central government of China together with local governments had tightened preventive measures. However, the virus had spread rapidly across most regions in China and in other countries and territories around the world. One major cause of the quick spread of COVID-19 was the lack of information and awareness about the virus during its early stages of infection. As of March 29, 2020, 13:29 GMT, COVID-19 has affected 199 countries and territories around the world with 683,536 confirmed cases, of which 32,139 have resulted in death and 25,422 are serious or critical (worldometers.info). However, there is still the possibility that the spread of this virus could become more intense and cause higher mortality. Chinese New Year celebrations accelerated the outbreak of COVID-19 throughout China, as many people travelled to their hometown or other destinations for the holiday.

Symptoms of COVID-19 take at least 2 to 10 days to develop, which makes it difficult to isolate infected individuals during the initial stages of the infection. The major symptoms of COVID-19 include dry cough and high fever with difficulties in breathing [3]. The virus may spread in the environment through respiratory droplets from infected individuals when they cough or sneeze. A previously unaffected populace may become infected when they are exposed to the virus by touching infected surfaces or while breathing in an infected environment [4]. During the initial stages of the COVID-19 outbreak, such human transmission was commonly taking place because the public were unaware of these risk factors, and infected individuals were not isolated, and were therefore spreading the virus unknowingly to other individuals. Moreover, the risk factor of contamination remained very high since the virus can remain viable in the environment for several days in favourable conditions $[5,6]$. Several studies have shown that the elderly, children, and those with serious diseases 
have lower immunity and tend to be seriously affected once they become infected $[7,8]$. At this time, we still lack any proper treatment or vaccines as a cure for the disease. Hence, to control its transmission further, isolating infected individuals in special quarantine arrangements has been implemented in many of the countries affected. Despite these preventative strategies, transmission is still ongoing and the mortality rate due to the virus remains at a high level.

To combat this situation, studies involving mathematical modelling play a crucial role in understanding the pandemic behaviour of infectious diseases. Bi et al. worked on stability and bifurcation of a mathematical model in the Zika virus. In addition, they established an optimal control problem including several popular disease intervention strategies which helped to mitigate against the Zika epidemics [9]. Zhao et al. modeled a vector-born visceral leishmaniasis disease transmission system, and in addition, they performed bifurcation analysis to support control conditions and analyse optimal control strategies to control zoonotic disease transmission [10]. Several studies have already been undertaken to analyse the COVID-19 transmission dynamics. Based on the database studies of COVID-19 outbreak from 31 December 2019 to 28 January 2020, Wu et al. introduced a SEIR model to estimate the spread of the disease nationally as well as globally [11]. Tang et al. proposed a compartmental model by dividing each group into two subpopulations, the quarantined and unquarantined. Moreover, they redesigned their previous model by using diagnosis and time-dependent contact rates and re-estimated the reproduction ratio to quantify the evolution in a better way $[12,13]$. Peng et al. designed a generalized SEIR model that suitably incorporates the intrinsic impact of hidden exposed and infectious cases of COVID-19 [14]. Chen et al. presented the incubation period behaviour of a local outbreak of COVID-19 by constructing a dynamic system [15]. Khan and Atangana described brief details of interaction among bats, unknown hosts, humans, and infection reservoirs by formulating the mathematical results of the mathematical fractional model [16]. Chen et al. have also developed a Bats-Hosts-ReservoirPeople transmission network model for simulating the potential transmission of COVID-19 [17]. Zhao et al. divided susceptible people from Wuhan city into different age groups and devel- oped a SEIARW model based on marketto-person and person-to-person transmission routes [18]. Zhong et al. constructed a mathematical model using epidemiological data and examined characteristics of the historical epidemic to make an early prediction of the COVID19 outbreak in China [19]. Yang and Wang have studied the multiple transmission pathways in the infection dynamics and formed a mathematical model that describes the role of the environmental reservoir in the transmission and spread of COVID-19 [20].

In this work, a COVID-19 model is constructed to study human-to-human transmission of the virus in Section 2. Optimal control theory is introduced and applied to the model for development in Section 3, and in Section 4, the model is simulated numerically to observe the effect of control strategies on the model.

\section{COVID-19 Model Formulation}

To analyse the human-to-human transmission dynamics of COVID-19, a compartmental model is constructed. The model consists of all possible human-to-human transmission dynamics of the virus. COVID-19 is highly contagious in nature, and cases have been seen in most of the countries around the world; hence, in the model, the susceptible population class is ignored and whole population is divided into five compartments: class of exposed individuals $E(t)$ (individuals surrounded by infection but not yet infected), class of infected individuals by COVID-19 $I(t)$, class of critically infected individuals by COVID-19 $C(t)$, class of hospitalised individuals $H(t)$, and class of dead individuals due to COVID-19 $D(t)$. Human-to-human transmission dynamics of COVID-19 are described graphically in Figure 1. Parameters used in the model are described in Table 1.

Using the above depiction, a dynamical system of set of nonlinear differential equations for the model is formulated as follows:

$$
\begin{aligned}
& \frac{\mathrm{d} E}{\mathrm{~d} t}=B-\beta_{1} E I+\beta_{7} E D+\beta_{9} H+\beta_{10} E I-\mu E, \\
& \frac{\mathrm{d} I}{\mathrm{~d} t}=\beta_{1} E I-\beta_{2} I-\beta_{6} I-\beta_{8} I-\beta_{10} E I-\mu I, \\
& \frac{\mathrm{d} C}{\mathrm{~d} t}=\beta_{2} I-\beta_{5} C-\beta_{3} C+\beta_{4} H-\mu C, \\
& \frac{\mathrm{d} H}{\mathrm{~d} t}=\beta_{3} C-\beta_{4} H+\beta_{8} I-\beta_{9} H-\mu H, \\
& \frac{\mathrm{d} D}{\mathrm{~d} t}=\beta_{5} C+\beta_{6} I-\beta_{7} D E .
\end{aligned}
$$

Note that all the parameters used in this COVID-19 model are nonnegative. Consider the feasible region as follows:

$$
\Lambda=\left\{(E, I, C, H, D) \in R_{+}^{5}: E+I+C+H+D \leq \frac{B}{\mu}\right\} .
$$

The region $\Lambda$ is positively invariant; all the solutions of system (1) remain in the feasible region (2).

2.1. Equilibrium Points. By solving system (1), we get two equilibrium points:

(i) Disease-free equilibrium point:

$$
E_{0}=\left(\frac{B}{\mu}, 0,0,0,0\right)
$$

(ii) Endemic equilibrium point:

$$
E^{*}=\left(\frac{\beta_{2}+\beta_{6}+\beta_{8}+\mu}{\beta_{1}-\beta_{10}}, \frac{i}{\mu q_{1}}, \frac{c}{\mu q_{1}}, \frac{h}{\mu q_{1}}, \frac{d}{\mu \beta_{7} q_{2}}\right),
$$

where 


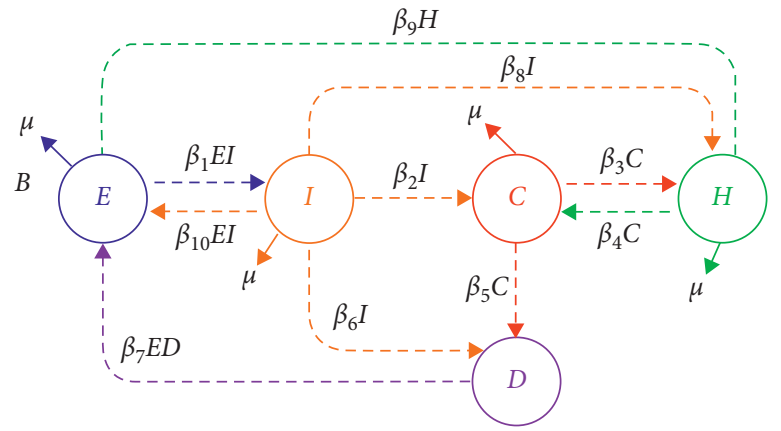

Figure 1: Transmission dynamics of COVID-19.

TABle 1: Parameters used in the model.

\begin{tabular}{|c|c|c|c|}
\hline \multicolumn{4}{|c|}{ Parameters } \\
\hline$B$ & Birth rate of class of exposed individuals & 0.80 & Calculated \\
\hline$\mu$ & Natural death rate & 0.01 & Assumed \\
\hline$\beta_{1}$ & Transmission rate of individuals moving from exposed to infected class & 0.55 & Calculated \\
\hline$\beta_{2}$ & Rate at which infected individuals go into severe condition or in critical condition & 0.40 & Calculated \\
\hline$\beta_{3}$ & Rate at which critically infected individuals get hospitalised & 0.60 & Calculated \\
\hline$\beta_{4}$ & Rate at which hospitalised individuals are not recovered and remain in critical condition & 0.80 & Calculated \\
\hline$\beta_{5}$ & Mortality rate of critically infected individuals & 0.34 & Calculated \\
\hline$\beta_{6}$ & Mortality rate of infected individuals & 0.30 & Calculated \\
\hline$\beta_{7}$ & Rate at which infected dead body spreads infection & 0.35 & Assumed \\
\hline$\beta_{8}$ & Rate at which infected individuals get hospitalised & 0.30 & Calculated \\
\hline$\beta_{9}$ & Rate at which hospitalised individuals get recovered and become exposed again & 0.35 & Assumed \\
\hline$\beta_{10}$ & Rate at which infected individuals recovered themselves due to strong immunity and again become exposed & 0.32 & Assumed \\
\hline
\end{tabular}

$$
\begin{aligned}
i= & \left(\beta_{5}+\mu\right)\left(B \beta_{4}\left(\beta_{1}-\beta_{10}\right)-\beta_{4} \mu\left(\beta_{2}+\beta_{6}\right)\right)-\left(\beta_{8}+\mu\right)\left(\beta_{5}+\mu\right) \beta_{4} \mu+\left(\beta_{9}+\mu\right)\left(\left(\beta_{3}+\beta_{5}\right)\left(B \beta_{1}-B \beta_{10}-\beta_{2} \mu\right)\right. \\
& \left.-\mu\left(\beta_{6}+\beta_{8}\right)\left(\beta_{3}+\beta_{5}+\mu\right)-B \mu\left(\beta_{1}+\beta_{10}\right)+\mu^{2}\left(\beta_{2}+\beta_{3}+\beta_{5}+\mu\right)\right) \\
c= & \left(\beta_{4}+\beta_{9}+\mu\right)\left(B \beta_{2}\left(\beta_{1}-\beta_{10}\right) \beta_{2}^{2} \mu\right)-\mu \beta_{4}\left(\beta_{8}+\mu\right)\left(\beta_{2}+\beta_{8}\right)-\beta_{4} \beta_{8}\left(B \beta_{1}-B \beta_{10}-\beta_{6} \mu\right)-\mu \beta_{2}\left(\beta_{9}+\mu\right)\left(1+\beta_{6}+\beta_{8}\right), \\
h= & \beta_{8}\left(\beta_{5}+\mu\right)\left(B \beta_{1}-B \beta_{10}-\beta_{2} \mu\right)-\beta_{8} \mu^{2}\left(\beta_{6}+\mu\right)-\mu \beta_{8}\left(\beta_{8}+\mu\right)\left(\beta_{3}+\beta_{5}\right)-\mu \beta_{6} \beta_{8}\left(\beta_{3}+\beta_{5}\right)-B \beta_{1} \beta_{2}\left(\beta_{3}+\beta_{8}\right) \\
& -B \beta_{10} \beta_{3}\left(\beta_{2}+\beta_{8}\right)-\mu \beta_{2} \beta_{3}\left(\beta_{2}+\beta_{6}\right)-\mu \beta_{8}\left(\beta_{2} \beta_{3}+\mu\right) \\
d= & \left(\beta_{9}+\mu\right)\left(B \beta_{6}\left(\beta_{1}-\beta_{10}\right)-\mu \beta_{6}^{2}\right)-\left(\beta_{8}+\mu\right)\left(\beta_{2} \beta_{5} \mu+\beta_{6} \mu\left(\beta_{3}+\beta_{5}+\beta_{6}+\mu\right)\right)-\beta_{2} \beta_{5} \mu\left(\beta_{2}+\beta_{6}\right)-\beta_{6} \mu\left(\beta_{2}\left(\beta_{3}+\mu\right)+\beta_{3} \beta_{6}\right) \\
& +B\left(\beta_{1}-\beta_{10}\right)\left(\beta_{3} \beta_{6}+\beta_{2} \beta_{5}\right)+\beta_{4} \beta_{6}\left(\beta_{5}+\mu\right)\left(B\left(\beta_{1}-\beta_{10}\right)-\mu\left(\beta_{2}+\beta_{8}\right)\right)-\mu \beta_{4}\left(\beta_{6}+\mu\right)\left(\beta_{2} \beta_{5}+\beta_{5} \beta_{6}+\beta_{6} \mu\right) \\
& -\left(\beta_{8}+\mu\right) \beta_{4} \beta_{5} \beta_{8} \mu-\beta_{4} \beta_{5}\left(\beta_{2}+\beta_{6}\right)\left(\mu\left(\beta_{8}+\beta_{2}\right)+B\left(\beta_{10}+\beta_{1}\right)\right) \\
q_{1}= & \left(\beta_{1}-\beta_{10}\right)\left(\beta_{2}\left(\beta_{4}+\beta_{9}+\mu\right)+\left(\beta_{8}+\beta_{9}+\mu\right)\left(\beta_{3}+\beta_{5}\right)+\beta_{4}\left(\beta_{5}+\beta_{8}+\mu\right)+\mu\left(\beta_{9}+\mu\right)+\beta_{2} \beta_{3}+\beta_{8} \mu\right), \\
q_{2}= & \mu \beta_{7}\left(\beta_{2}+\beta_{6}+\beta_{8}\right)\left(\beta_{2} \beta_{3}+\beta_{2}\left(\beta_{4}+\beta_{8}+\mu\right)+\beta_{4}\left(\beta_{5}+\beta_{8}+\mu\right)+\left(\beta_{8}+\beta_{9}+\mu\right)\left(\beta_{3}+\beta_{5}+\mu\right)\right) .
\end{aligned}
$$

2.2. Basic Reproduction Number. The basic reproduction number $\left(R_{0}\right)$ for the model can be established using the next-generation matrix method $[21,22]$. The basic reproduction number $\left(R_{0}\right)$ is obtained as the spectral radius of matrix $\left(F V^{-1}\right)$ at the disease-free equilibrium point, where $F$ and $V$ are as follows: 
$F=\left[\begin{array}{ccccc}0 & \frac{B \beta_{10}}{\mu} & 0 & 0 & \frac{B \beta_{7}}{\mu} \\ 0 & \frac{B \beta_{1}}{\mu} & 0 & 0 & 0 \\ 0 & 0 & 0 & 0 & 0 \\ 0 & 0 & 0 & 0 & 0 \\ 0 & 0 & 0 & 0 & 0\end{array}\right]$,

$V=\left[\begin{array}{ccccc}\mu & \frac{B \beta_{1}}{\mu} & 0 & -\beta_{9} & 0 \\ 0 & \frac{B \beta_{10}}{\mu}+\beta_{8}+\beta_{6}+\beta_{2}+\mu & 0 & 0 & 0 \\ 0 & -\beta_{2} & \beta_{3}+\beta_{5}+\mu & -\beta_{4} & 0 \\ 0 & -\beta_{8} & -\beta_{3} & \beta_{9}+\beta_{4}+\mu & 0 \\ 0 & -\beta_{6} & -\beta_{5} & 0 & \frac{B \beta_{7}}{\mu}\end{array}\right]$.

The defined effective basic reproduction number is

$$
R_{0}=\frac{B \beta_{1}}{B \beta_{10}+\mu\left(\beta_{2}+\beta_{6}+\beta_{8}+\mu\right)} .
$$

\section{Optimal Control Theory}

Control measures can play a significant role in controlling the epidemic of COVID-19 at a certain level. In this control theory, five control variables are used as five possible control strategies. Since the virus is highly contagious, it quickly infects any people who come into contact with an infected individual. To avoid this situation, we have included $u_{1}$ as a control variable for self-quarantine of exposed individuals and $u_{2}$ as a control variable for isolation of infected individuals. Moreover, to minimise the mortality rate of COVID-19, $u_{3}$ control variable is included which helps to reduce critically infected cases by taking extra medical care of infected individuals. $u_{4}$ and $u_{5}$ control variables are included to improve hospitalization facilities for infected and critically infected individuals, respectively. The purpose of this study of control theory is to protect people from the outbreak by applying controls or treatment in each stage. The objective function for the required scenario is

$$
\begin{aligned}
J\left(c_{i}, \Omega\right)= & \int_{0}^{T}\left(A_{1} E^{2}+A_{2} I^{2}+A_{3} C^{2}+A_{4} H^{2}+A_{5} D^{2}\right. \\
& \left.+w_{1} u_{1}^{2}+w_{2} u_{2}^{2}+w_{3} u_{3}^{2}+w_{4} u_{4}^{2}+w_{5} u_{5}^{2}\right) \mathrm{d} t
\end{aligned}
$$

where $\Omega$ denotes the feasible region for the set of compartmental variables, $A_{1}, A_{2}, A_{3}, A_{4}$, and $A_{5}$ denote the nonnegative weight constants for compartments $E, I, C, H$, and $D$, respectively. $w_{1}, w_{2}, w_{3}, w_{4}$, and $w_{5}$ are the weight constants for each control $u_{i}$, where $i=1,2, \ldots, 5$, respectively. The modified model is given in Figure 2.

Hence, the modified system for Figure 2 is stated as follows:

$$
\begin{aligned}
& \frac{\mathrm{d} E}{\mathrm{~d} t}=B-\beta_{1} E I+\beta_{7} E D+\beta_{9} H+\beta_{10} E I-u_{1} E+u_{2} I-\mu E, \\
& \frac{\mathrm{d} I}{\mathrm{~d} t}=\beta_{1} E I-\beta_{2} I-\beta_{6} I-\beta_{8} I-\beta_{10} E I+u_{1} E-u_{2} I-u_{5} I-u_{3} I-\mu I, \\
& \frac{\mathrm{d} C}{\mathrm{~d} t}=\beta_{2} I-\beta_{5} C-\beta_{3} C+\beta_{4} H+u_{3} I-u_{4} C-\mu C, \\
& \frac{\mathrm{d} H}{\mathrm{~d} t}=\beta_{3} C-\beta_{4} H+\beta_{8} I-\beta_{9} H+u_{5} I+u_{4} C-\mu H, \\
& \frac{\mathrm{d} D}{\mathrm{~d} t}=\beta_{5} C+\beta_{6} I-\beta_{7} D E .
\end{aligned}
$$

The weight parameters $w_{i}, i=1,2, \ldots, 5$, are constants applied on the control variable, respectively, from which the optimal condition is normalized. Now, every value of control variables is calculated from $t=0$ to $t=T$ such that

$$
J\left(u_{i}(t)\right)=\min \left\{J\left(u_{i}^{*}, \Omega\right) /\left(u_{i}\right) \in \phi\right\}, \quad i=1,2, \ldots, 5,
$$

where $\phi$ is a smooth function on the interval $[0,1]$. The optimal effect is found by using the results of Fleming and Lions [23]. We use Pontryagin's Maximum Principle to derive the optimal amount of control [24]. The associated Lagrangian function with adjoint variables $\lambda_{1}, \lambda_{2}, \lambda_{3}, \lambda_{4}$, and $\lambda_{5}$ is given by

$$
\begin{aligned}
L(\Omega, A)= & A_{1} E^{2}+A_{2} I^{2}+A_{3} C^{2}+A_{4} H^{2}+A_{5} D^{2}+w_{1} u_{1}^{2} \\
& +w_{2} u_{2}^{2}+w_{3} u_{3}^{2}+w_{4} u_{4}^{2}+w_{5} u_{5}^{2} \\
& +\lambda_{1}\left(B-\beta_{1} E I+\beta_{7} E D+\beta_{9} H+\beta_{10} E I-\mu E\right) \\
& +\lambda_{2}\left(\beta_{1} E I-\beta_{2} I-\beta_{6} I-\beta_{8} I-\beta_{10} E I-\mu I\right) \\
& +\lambda_{3}\left(\beta_{2} I-\beta_{5} C-\beta_{3} C+\beta_{4} H-\mu C\right) \\
& +\lambda_{4}\left(\beta_{3} C-\beta_{4} H+\beta_{8} I-\beta_{9} H-\mu H\right) \\
& +\lambda_{5}\left(\beta_{5} C+\beta_{6} I-\beta_{7} D E\right) .
\end{aligned}
$$

The partial derivatives of the Lagrangian function with respect to each variable of the compartment give the adjoint equation variables $A_{i}=\left(\lambda_{1}, \lambda_{2}, \lambda_{3}, \lambda_{4}, \lambda_{5}\right)$ corresponding to the system which are as follows: 


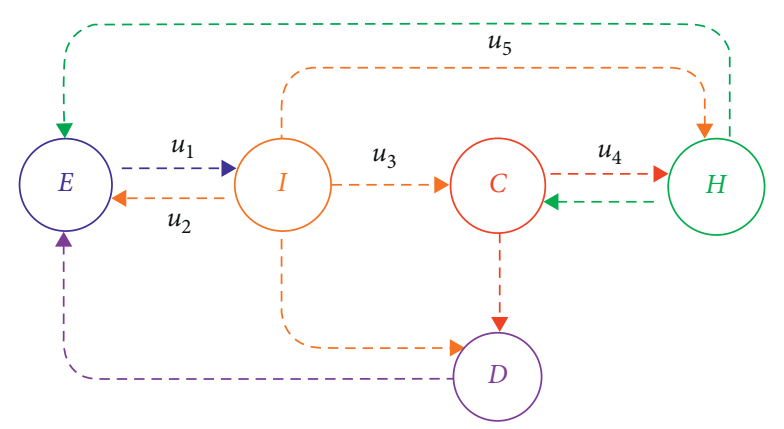

Figure 2: COVID-19 model with control variables.

$$
\begin{aligned}
\dot{\lambda_{1}}= & -\frac{\partial L}{\partial E}=-2 A_{1} E+\left(\lambda_{1}-\lambda_{2}\right) \beta_{1} I-\left(\lambda_{1}-\lambda_{2}\right) \beta_{10} I \\
& -\lambda_{1} \beta_{7} \mathrm{D}-\lambda_{1} \mu+\lambda_{5} \beta_{7} \mathrm{D}+\left(\lambda_{1}-\lambda_{2}\right) u_{1}, \\
\dot{\lambda_{2}}= & -\frac{\partial L}{\partial I}=-2 A_{2} I+\left(\lambda_{1}-\lambda_{2}\right)\left(\beta_{1} E-\beta_{10} E-u_{2}\right) \\
& +\left(\lambda_{2}-\lambda_{3}\right)\left(\beta_{2}+u_{3}\right)+\left(\lambda_{2}-\lambda_{4}\right)\left(\beta_{8}+u_{5}\right)+\left(\lambda_{2}-\lambda_{5}\right) \beta_{6}+\lambda_{2} \mu,
\end{aligned}
$$$$
\dot{\lambda}_{3}=-\frac{\partial L}{\partial C}=-2 A_{3} C+\left(\lambda_{3}-\lambda_{4}\right)\left(\beta_{3}+u_{4}\right)+\left(\lambda_{3}-\lambda_{5}\right) \beta_{5}+\lambda_{3} \mu,
$$$$
\dot{\lambda}_{4}=-\frac{\partial L}{\partial H}=-2 A_{4} H-\left(\lambda_{1}-\lambda_{4}\right) \beta_{9}-\left(\lambda_{3}-\lambda_{4}\right) \beta_{4}+\lambda_{4} \mu,
$$$$
\dot{\lambda}_{5}=-\frac{\partial L}{\partial D}=-2 A_{5} D-\left(\lambda_{1}-\lambda_{5}\right) \beta_{7} E .
$$

Hence, the optimal controls are given by

$$
\begin{aligned}
& u_{1}=\frac{\left(\lambda_{1}-\lambda_{2}\right) E}{2 w_{1}}, \\
& u_{2}=\frac{\left(\lambda_{2}-\lambda_{1}\right) I}{2 w_{2}}, \\
& u_{3}=\frac{\left(\lambda_{2}-\lambda_{3}\right) I}{2 w_{3}}, \\
& u_{4}=\frac{\left(\lambda_{3}-\lambda_{4}\right) C}{2 w_{4}}, \\
& u_{5}=\frac{\left(\lambda_{2}-\lambda_{4}\right) I}{2 w_{5}} .
\end{aligned}
$$

In addition, the optimal conditions are given as follows:

$$
\begin{aligned}
& u_{1}^{*}=\max \left(a_{1}, \min \left(b_{1}, \frac{\left(\lambda_{1}-\lambda_{2}\right) E}{2 w_{1}}\right)\right), \\
& u_{2}^{*}=\max \left(a_{2}, \min \left(b_{2}, \frac{\left(\lambda_{2}-\lambda_{1}\right) I}{2 w_{2}}\right)\right), \\
& u_{3}^{*}=\max \left(a_{3}, \min \left(b_{3}, \frac{\left(\lambda_{2}-\lambda_{3}\right) I}{2 w_{3}}\right)\right), \\
& u_{4}^{*}=\max \left(a_{4}, \min \left(b_{4}, \frac{\left(\lambda_{3}-\lambda_{4}\right) C}{2 w_{4}}\right)\right), \\
& u_{5}^{*}=\max \left(a_{5}, \min \left(b_{5}, \frac{\left(\lambda_{2}-\lambda_{4}\right) I}{2 w_{5}}\right)\right) .
\end{aligned}
$$

This calculation provides analytical behaviour of optimal control on the system. Numerical interpretation of optimal control theory is simulated in the next section.

\section{Numerical Simulation}

In this section, the COVID-19 model is simulated numerically, wherein the parametric values for simulation are taken from the recent pandemic outbreak of coronavirus (https://www.who.int/emergencies/diseases/novel-coronavirus2019/situation-reports). The initial condition taken to plot variations in all the compartments is $y_{0}(E, I, C, H, D)=$ $(8,4,0.12,1,5)$.

Figure 3 represents the variations in all the compartments of the COVID-19 model with respect to time. In addition, the pandemic behaviour of the COVID-19 outbreak can be clearly seen here. We can say that a large population of exposed individuals become infected within the first week. Moreover, the critically infected cases and hospitalization cases also increase with time. Furthermore, it clearly shows that after one week, the mortality rate is also increased.

Figure 4(a) shows variation in each compartment with and without control variables. It is observed that the 


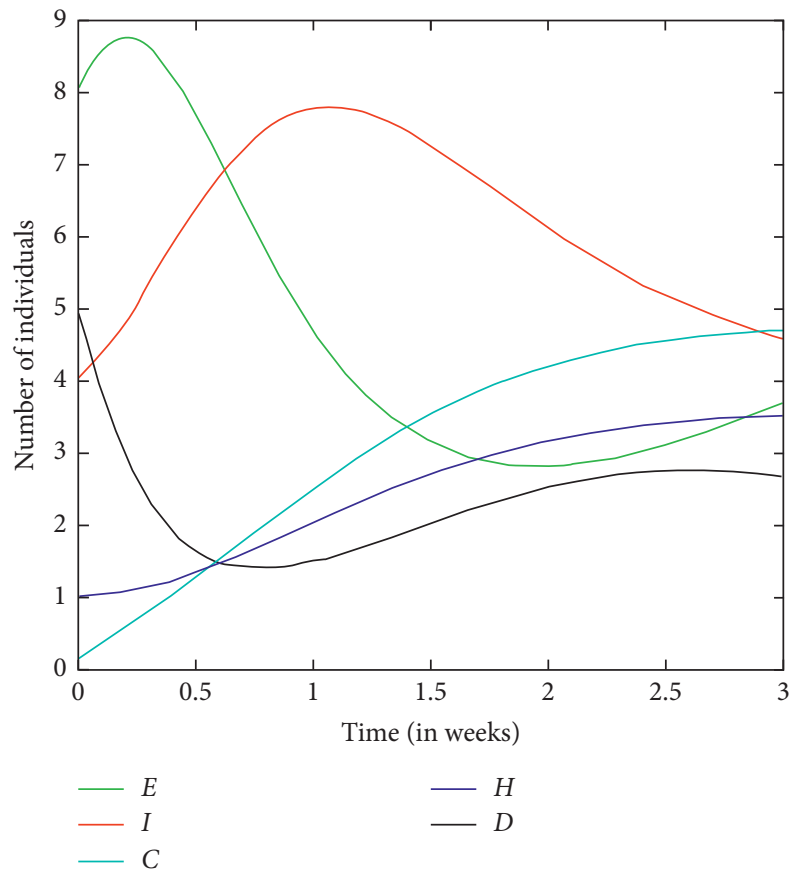

FIgURE 3: Variations in all compartments.

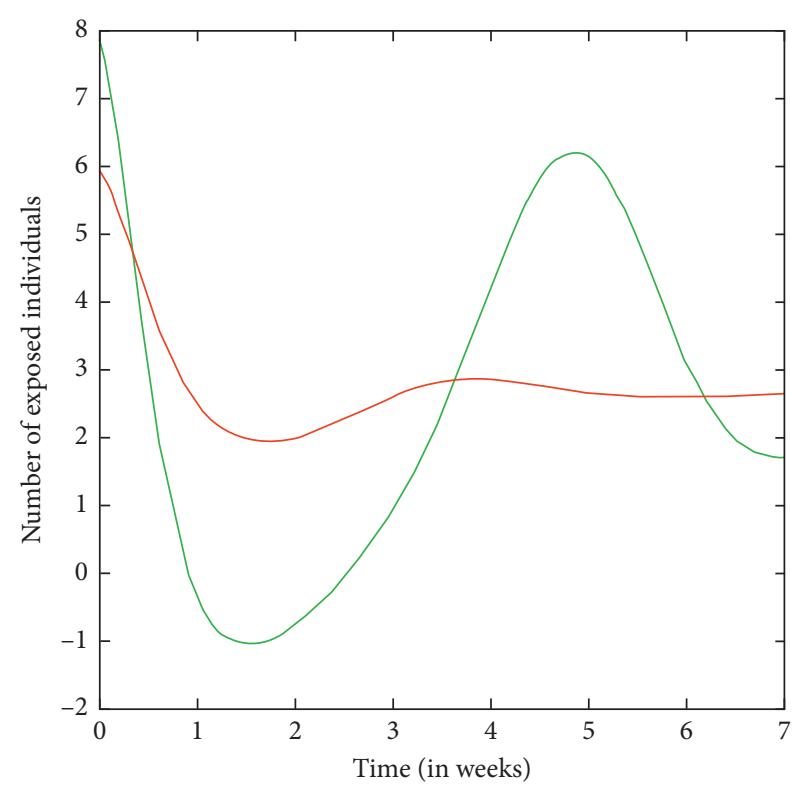

With all controls

Without controls

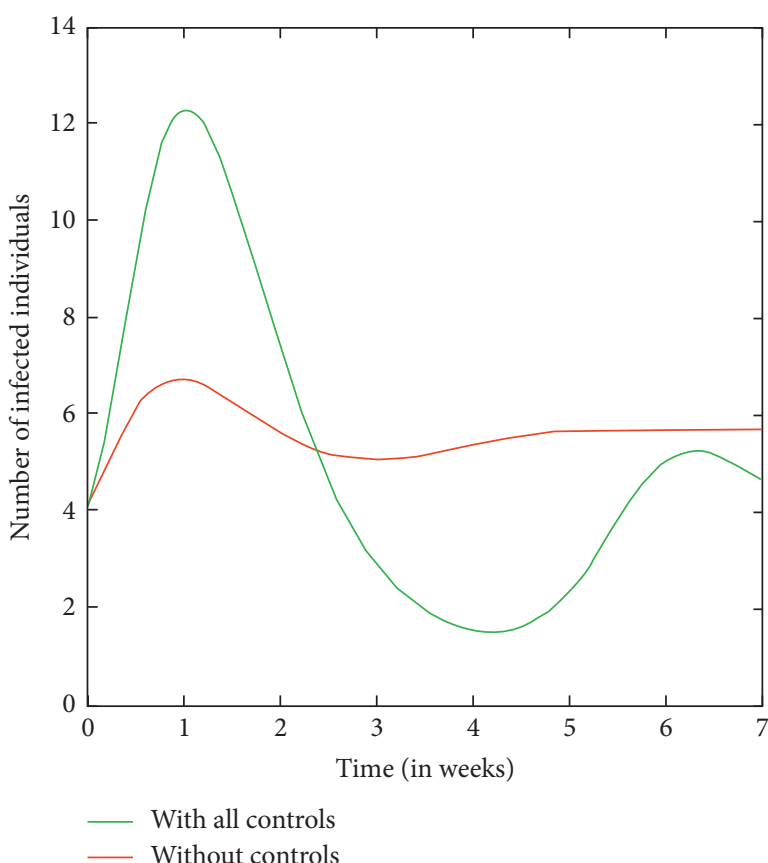

(b)

FIgURe 4: Continued. 


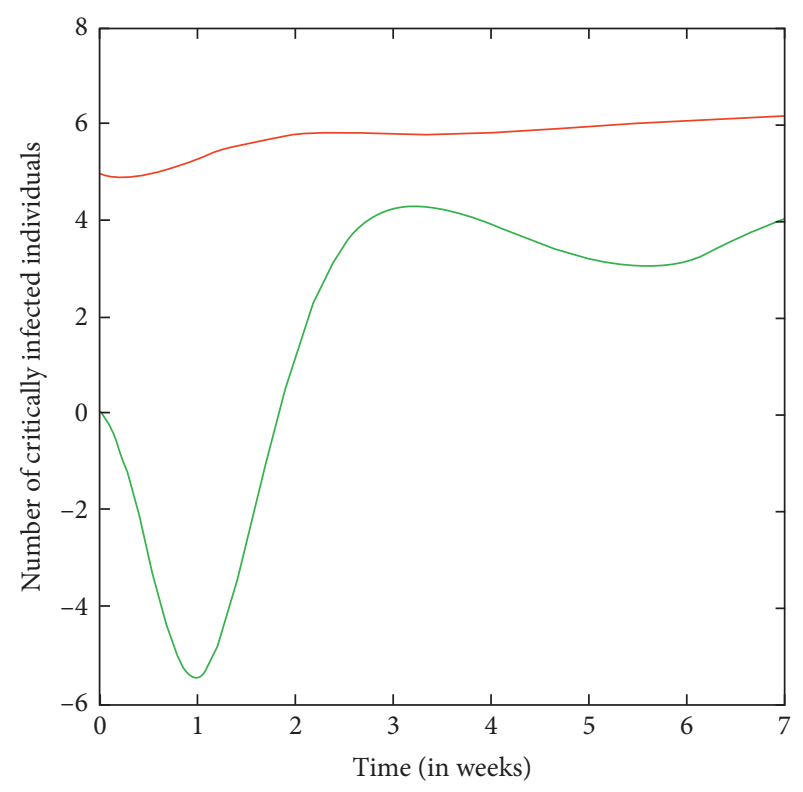

W With all controls Without controls

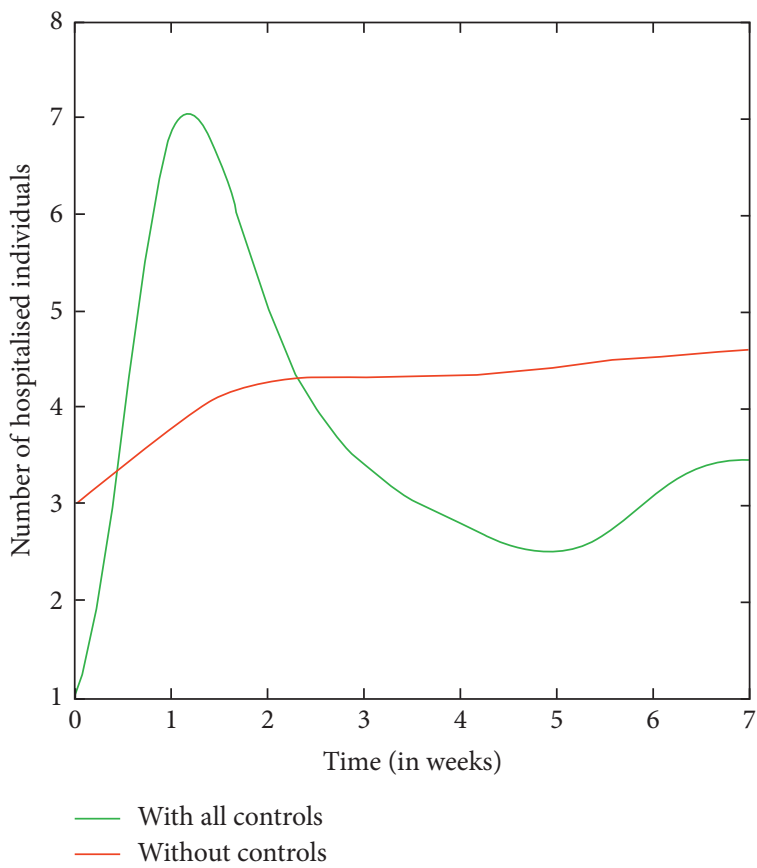

(d)

(c)

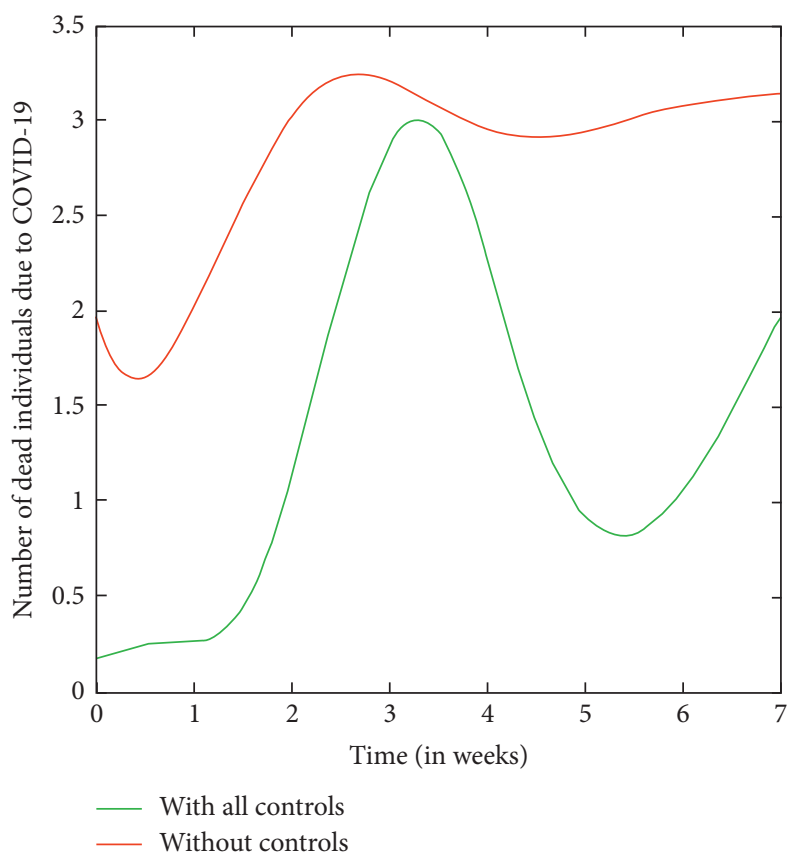

(e)

FIGURE 4: Variation in compartments with and without optimal controls.

COVID-19 outbreak can be controlled up to a certain level in seven weeks after applying control strategies. Meanwhile, Figures 4(b) and 4(c) represent the number of infected and critically-infected individuals decreasing; this then leads to a decrease in the hospitalized class as shown in Figure 4(d). Thus, reduction in the mortality rate under the effect of control strategies can be clearly observed Figure 4(e).
Variation in the intensity of control strategies with time is shown in Figure 5. Moreover, the figure also demonstrates which control can be applied and at what intensity to control a COVID-19 outbreak in seven weeks. The range of all the four controls $u_{1}, u_{2}, u_{3}$, and $u_{4}$ is [-0.7481, 0.4776], $[-1.733,0.8913],[-7.409,1.04]$, and $[0.0158,0.6338]$, respectively. The high fluctuation in the $u_{3}$ control variable at an initial stage suggests that it is very important to control 


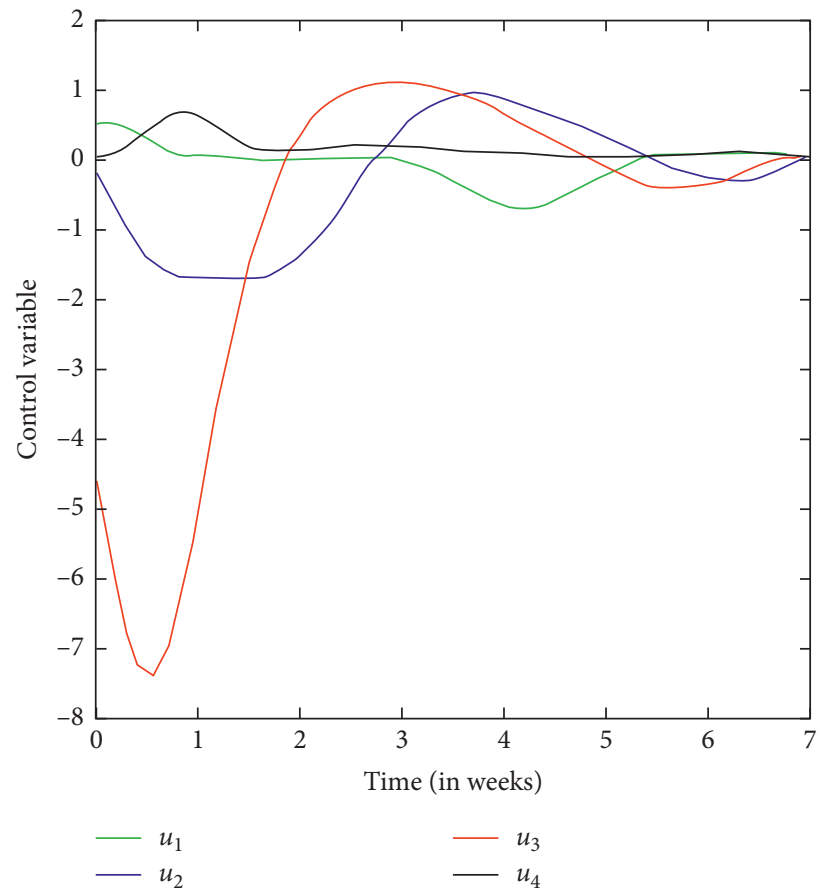

FIGURE 5: Variation in control variable with time.
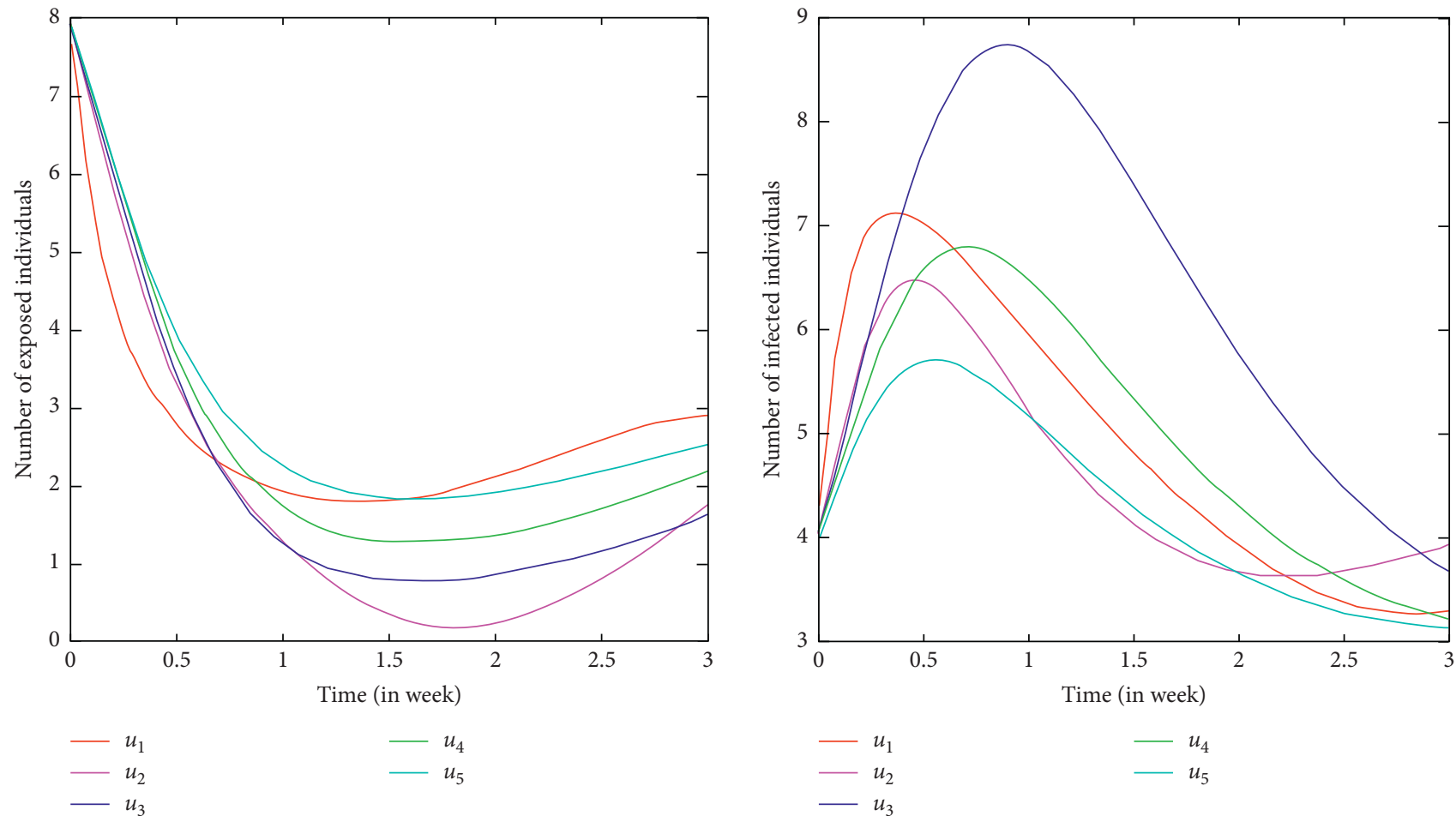

(a)

Figure 6: Continued.

(b) 


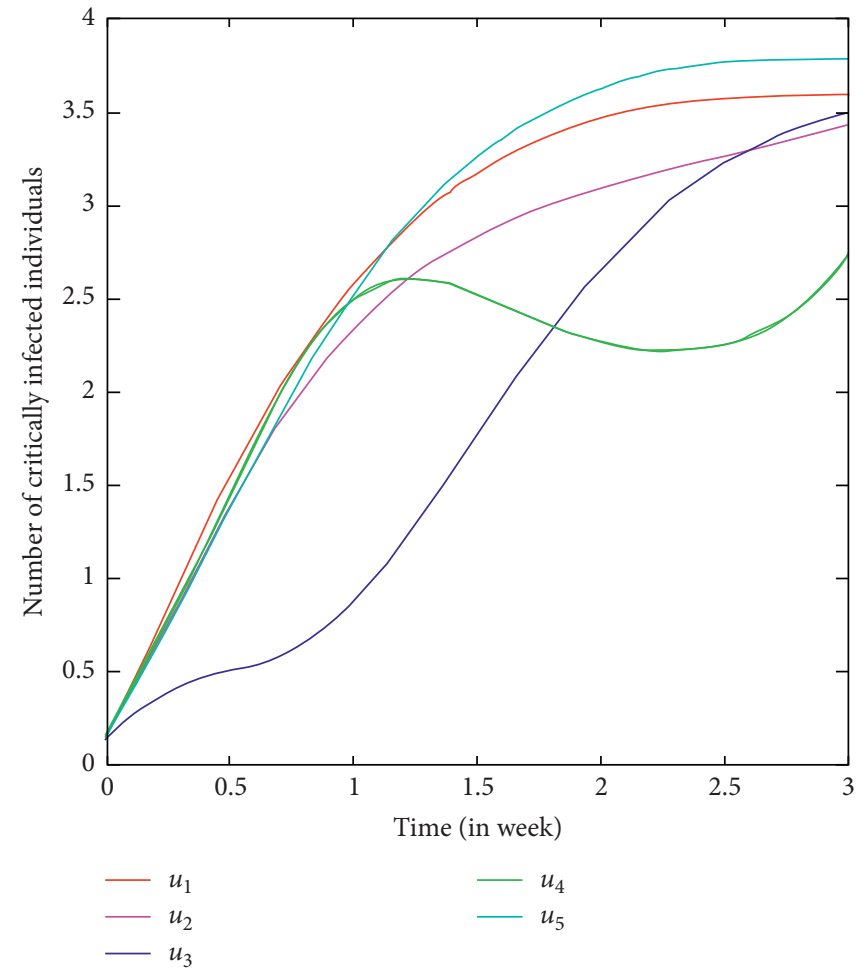

(c)

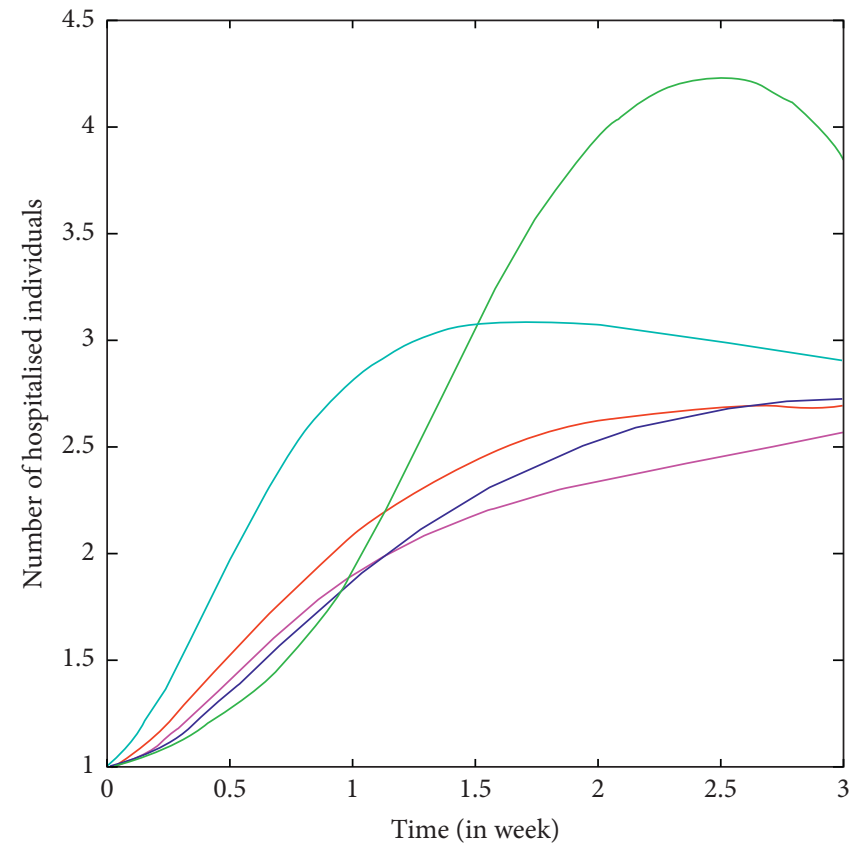

$-u_{1}$
$-u_{2}$
$-u_{3}$

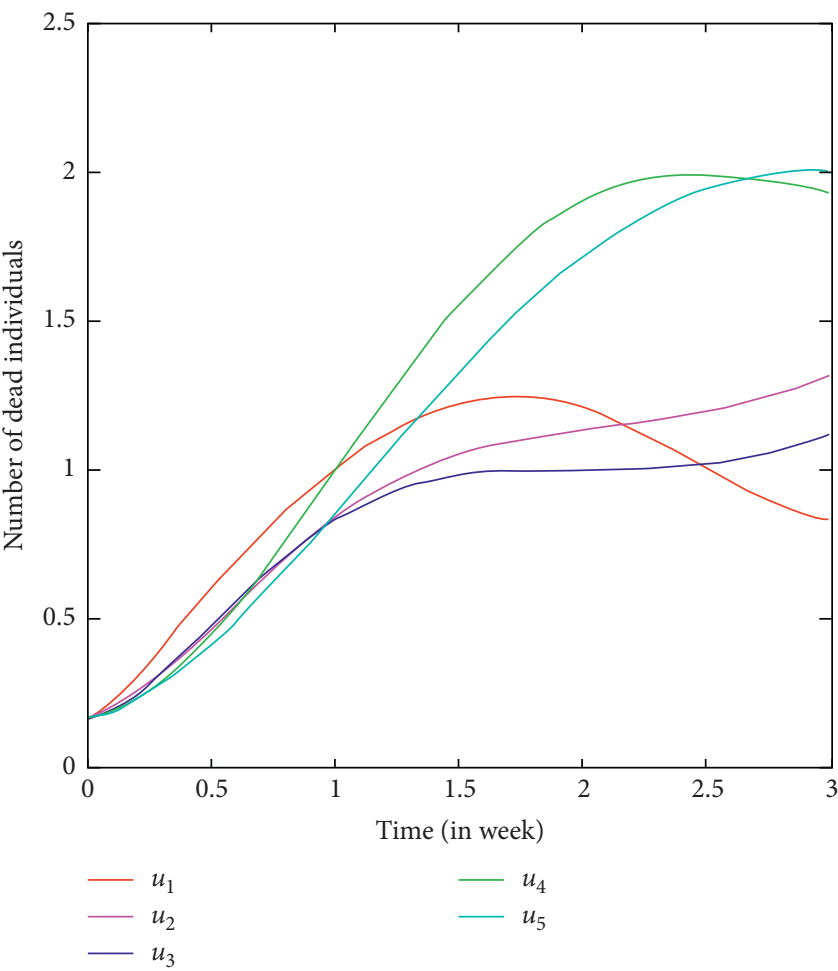

(e)

FIgURE 6: Variation in each compartment under individual effect of control variables.

infected individuals moving to the critical stage to reduce the mortality rate due to COVID-19. In addition, this could be achieved easily if an infected individual gets proper vaccination for the disease. Since effective vaccination is not available for COVID-19, proper care of infected individuals must be taken to improve their immunity so that their body 


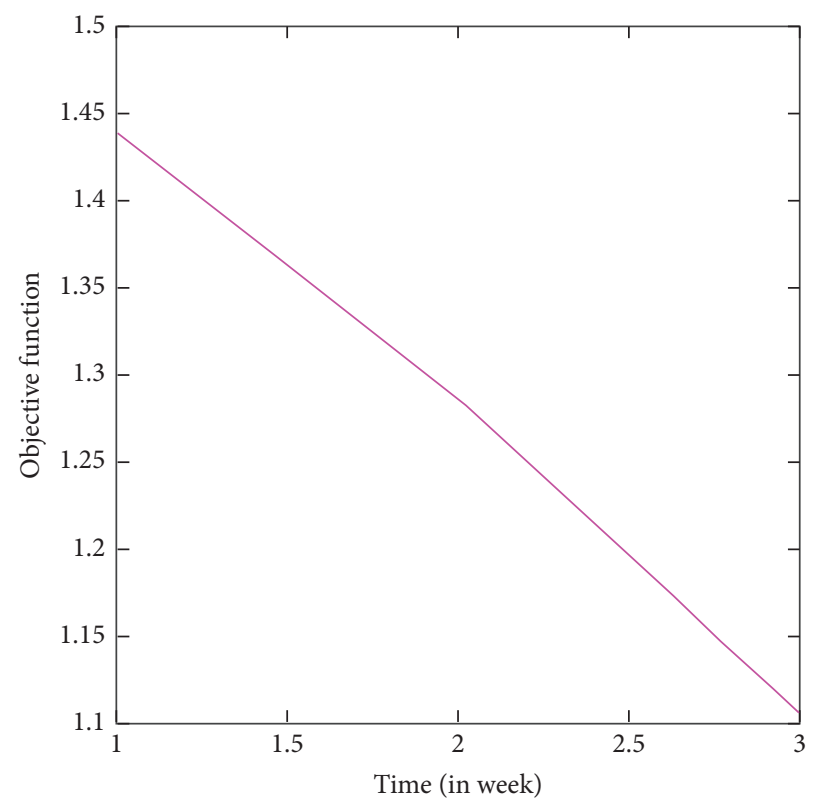

FIGURE 7: Change in objective function with time.

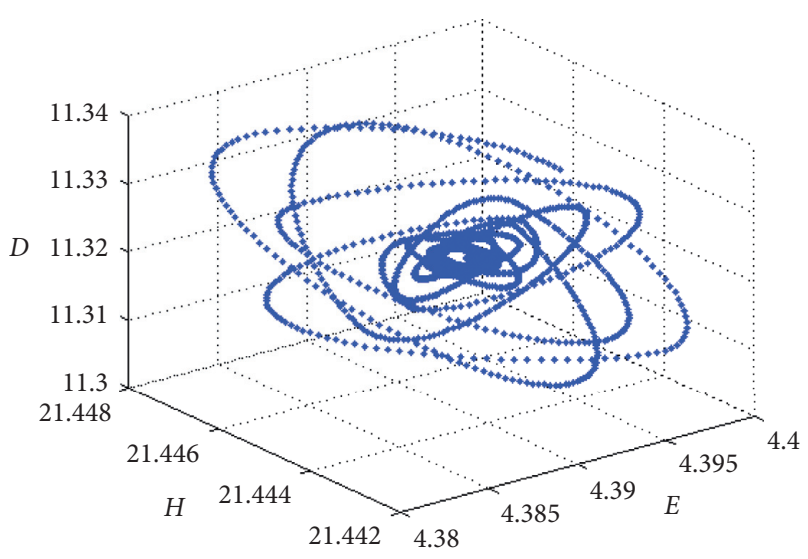

(a)

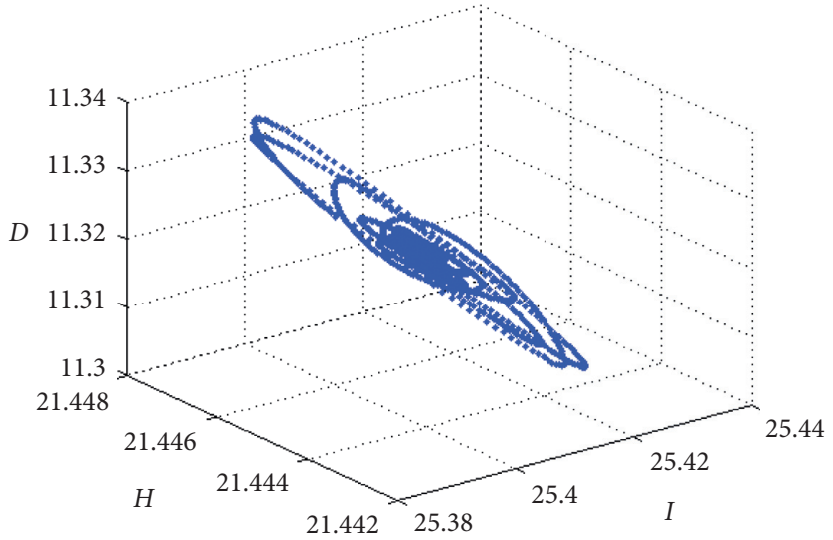

(b)

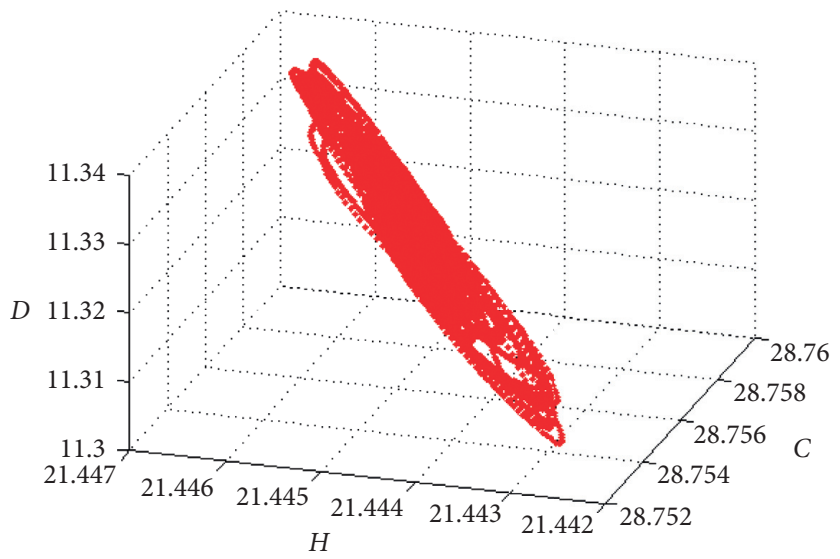

(c)

FIgURE 8: Scatter diagram for COVID-19 outbreak. 
becomes capable of fighting the virus and they do not reach the critical stage. Moreover, fluctuation in the $u_{2}$ control variable suggests that it is very important to isolate or quarantine infected individuals to control this pandemic outbreak.

The separate effects of control variables on each compartment can be observed in Figure 6. From this figure, we can interpret that the $u_{2}$ control variable is highly effective for stabilizing the epidemic situation. Figure 6(b) shows that the population class of infected individuals is lowest under the influence of the $u_{5}$ control variable which suggests that rapid hospitalization of infected individuals is an effective measure for reducing infected cases of COVID-19. Figure 6(c) suggests that in order to reduce critical cases of COVID-19,, we should first implement controls to prevent infected individuals from becoming critically infected, and that we should improve hospitalization and medical facilities for these critically infected individuals to save their lives. Figure 6(e) shows that the mortality rate due to COVID-19 can be reduced effectively within three weeks of outbreak by applying $u_{1}, u_{2}$, and $u_{3}$ control strategies. This means that self-quarantine for an exposed individual, isolation of an infected individual, and reducing critical cases by taking extra care of infected individuals are likely to be effective strategies to control further transmission of COVID-19.

Figure 7 represents the change in the objective function under influence of all the controls. The combined effect of all the controls indicates a desirable effect on the model.

Figure 8 demonstrates the scatter diagram representing the chaotic situation created during a COVID-19 outbreak. Figures $8(a)-8(c)$ show periodic mortality from classes of exposed individuals, infected individuals, and critically infected individuals with respect to time (in weeks), respectively, when under hospitalization. By comparing Figures 8(a) and 8(b), it can be interpreted that the mortality ratio in the class of infected individuals is higher and much faster than that for the class of exposed individuals. The chaotic Figure 8(c) diagram represents a very high mortality rate for critically infected individuals. Hence, in the absence of an effective vaccination for COVID-19, it becomes a challenging situation to cure critically infected individuals.

\section{Conclusion}

In this study, a compartmental model is constructed to examine transmission of COVID-19 within the human population. Moreover, the basic reproduction number is formulated to calculate the threshold value of the disease. In order to develop strategies to counteract COVID-19 epidemics, optimal control theory is applied to the model. In addition, in order to advance this control theory, five control variables are introduced in the model in the form of control strategies. These strategies include self-quarantine of exposed individuals, isolation of infected individuals, taking extra care of infected individuals to reduce critical cases of COVID-19, and increased hospitalization facilities for infected and critically infected individuals. Individual and combined effects of these control variables on all the compartments are observed and examined graphically by simulating the COVID-19 model. Numerical simulation of the model indicates that quarantine and better medical treatment of infected individuals can reduce the number of critically-infected cases, which will further reduce the transmission risk and mortality rate.

\section{Data Availability}

The data used to support the findings of this study are included within this article.

\section{Conflicts of Interest}

The authors declare that they have no conflicts of interest.

\section{Acknowledgments}

The authors are thankful to DST-FIST file \# MSI-097 for technical support to the Department of Mathematics, Gujarat University. AHS is funded by a Junior Research Fellowship from the Council of Scientific \& Industrial Research (file no. 09/070(0061)/2019-EMR-I). ENJ is funded by UGC granted National Fellowship for Other Backward Classes (NFO-2018-19-OBC-GUJ-71790).

\section{References}

[1] H. A. Rothan and S. N. Byrareddy, "The epidemiology and pathogenesis of coronavirus disease (COVID-19) outbreak," Journal of Autoimmunity, vol. 109, Article ID 102433, 2020.

[2] C. Sohrabi, Z. Alsafi, N. O'Neill et al., "World Health Organization declares global emergency: a review of the 2019 novel coronavirus (COVID-19)," International Journal of Surgery, vol. 76, pp. 71-76, 2020.

[3] I. Thevarajan, T. H. Nguyen, M. Koutsakos et al., "Breadth of concomitant immune responses prior to patient recovery: a case report of non-severe COVID-19," Nature Medicine, pp. 1-3, 2020.

[4] J. Riou and C. L. Althaus, "Pattern of early human-to-human transmission of Wuhan 2019 novel coronavirus (2019-nCoV), December 2019 to January 2020," Eurosurveillance, vol. 25, no. $4,2020$.

[5] A. R. Sahin, A. Erdogan, P. M. Agaoglu et al., "2019 novel coronavirus (COVID-19) outbreak: a review of the current literature," EJMO, vol. 4, no. 1, pp. 1-7, 2020.

[6] Z. J. Cheng and J. Shan, "2019 Novel coronavirus: where we are and what we know," Infection, vol. 48, no. 2, pp. 1-9, 2020.

[7] W. Y. Khot and M. Y. Nadkar, "The 2019 novel coronavirus outbreak-a global threat," The Journal of the Association of Physicians of India, vol. 68, no. 3, pp. 67-71, 2020.

[8] Wang, Coronavirus Disease 2019 (COVID-19): Situation Report, WHO, Geneva, Switzerland, 2020.

[9] K. Bi, Y. Chen, C.-H. Wu, and D. Ben-Arieh, "A memetic algorithm for solving optimal control problems of Zika virus epidemic with equilibriums and backward bifurcation analysis," Communications in Nonlinear Science and Numerical Simulation, vol. 84, p. 105176, 2020.

[10] S. Zhao, Y. Kuang, C. H. Wu, D. Ben-Arieh, M. RamalhoOrtigao, and $\mathrm{K} . \mathrm{Bi}$, "Zoonotic visceral leishmaniasis transmission: modeling, backward bifurcation, and optimal control," Journal of Mathematical Biology, vol. 73, no. 6-7, pp. 1525-1560, 2016. 
[11] J. T. Wu, K. Leung, and G. M. Leung, "Nowcasting and forecasting the potential domestic and international spread of the 2019-nCoV outbreak originating in Wuhan, China: a modelling study," The Lancet, vol. 395, no. 10225, pp. 689697, 2020.

[12] B. Tang, N. L. Bragazzi, Q. Li, S. Tang, Y. Xiao, and J. Wu, "An updated estimation of the risk of transmission of the novel coronavirus (2019-nCov)," Infectious Disease Modelling, vol. 5, pp. 248-255, 2020.

[13] B. Tang, X. Wang, Q. Li et al., "Estimation of the transmission risk of the 2019-nCoV and its implication for public health interventions," Journal of Clinical Medicine, vol. 9, no. 2, p. 462, 2020.

[14] L. Peng, W. Yang, D. Zhang, C. Zhuge, and L. Hong, "Epidemic analysis of COVID-19 in China by dynamical modelling," 2020, http://arxiv.org/abs/2002.06563.

[15] Y. Chen, J. Cheng, Y. Jiang, and K. Liu, "A time delay dynamic system with external source for the local outbreak of 2019nCoV," Applicable Analysis, pp. 1-12, 2020.

[16] M. A. Khan and A. Atangana, "Modeling the dynamics of novel coronavirus (2019-nCov) with fractional derivative," Alexandria Engineering Journal, pp. 1-11, 2020.

[17] T. Chen, J. Rui, Q. Wang, Z. Zhao, J. A. Cui, and L. Yin, “A mathematical model for simulating the transmission of Wuhan novel coronavirus," 2020.

[18] Z. Zhao, Y. Z. Zhu, J. W. Xu et al., "A mathematical model for estimating the age-specific transmissibility of a novel coronavirus," Infectious Diseases of Poverty, vol. 9, p. 24, 2020.

[19] L. Zhong, L. Mu, J. Li, J. Wang, Z. Yin, and D. Liu, "Early prediction of the 2019 novel coronavirus outbreak in the mainland China based on simple mathematical model," IEEE Access, vol. 8, pp. 51761-51769, 2020.

[20] C. Yang, "A mathematical model for the novel coronavirus epidemic in Wuhan, China," Mathematical Biosciences and Engineering, vol. 17, no. 3, pp. 2708-2724, 2020.

[21] O. Diekmann, J. A. P. Heesterbeek, and J. A. Metz, "On the definition and the computation of the basic reproduction ratio $R_{0}$ in models for infectious diseases in heterogeneous populations," Journal of Mathematical Biology, vol. 28, no. 4, pp. 365-382, 1990.

[22] P. Driessche and J. Watmough, "Reproduction numbers and sub-threshold endemic equilibria for compartmental models of disease transmission," Mathematical Biosciences, vol. 180, no. 1, pp. 29-48, 2002.

[23] W. Fleming and P. L. Lions, "Stochastic differential systems, stochastic control theory and applications: proceedings of a workshop, held at IMA, June 9-19, 1986," Springer Science \& Business Media, vol. 10, 2012.

[24] L. S. Pontryagin, V. G. Boltyanskij, R. V. Gamkrelidze, and E. F. Mishchenko, The Mathematical Theory of Optimal Processes, John Wiley \& Sons, New York, NY, USA, 1962. 